\title{
EL HOSPITAL DE SANTA MARTA DE SEVILLA: ACTIVIDADES Y GESTIÓN ECONÓMICA (SIGLOS XV A XVIII)
}

\author{
POR \\ José Julián Hernández Borreguero \\ Universidad de Sevilla
}

\section{RESUMEN}

Durante más de cuatro siglos el Hospital de Santa Marta de Sevilla desempeñó la función que le encomendó su fundador en 1404: dar alimento a los pobres. Para ello contaba con la propiedad de una serie de bienes inmuebles que arrendaba y dotaciones económicas que le permitían desarrollar holgadamente sus fines. Toda su gestión económica era tutelada y supervisada por el Cabildo Catedral hispalense. En este artículo se analizan las actividades realizadas por esta entidad a partir de los registros contables conservados sobre su gestión económica.

PALABRAS ClAVE: Patronato, hospital, caridad cristiana, alimento.

\section{THE SANTA MARTA HOSPITAL OF SEVILLE: ACTIVITIES AND ECONOMIC MANAGEMENT $\left(15^{\mathrm{TH}}\right.$ UNTIL $18^{\mathrm{TH}}$ CENTURIES $)$}

\begin{abstract}
During more than four centuries the Santa Marta Hospital of Seville developed the aim of his founder in 1404: give food to the poor. For this the Hospital rented their properties and could achieve its targets. All the economic process was supervised by the Cathedral Council of Seville. In this article the activities of this entity are analyzed through its accounting books.
\end{abstract}

KEY WORDS: Patronage, hospital, Christian Charity, food.

$$
\begin{array}{ll}
\text { Recibido/Received } & \text { 15-11-2013 } \\
\text { Aceptado/Accepted } & \text { 15-12-2014 }
\end{array}
$$




\section{INTRODUCCIÓN}

El concepto de caridad está íntimamente sujeto al Cristianismo desde su fundación, y fue recogido como tal en los Evangelios. ${ }^{1}$ La Iglesia consideró en la Antigüedad la atención a los enfermos y pobres como un medio que permitía a los hombres la salvación de sus almas. ${ }^{2}$

Esta virtud teologal se institucionalizó en el mundo romano, probablemente cuando el emperador Constantino prohíbe al arte curativo del sacerdocio pagano, especializados en la asistencia de enfermedades físicas y mentales, ${ }^{3}$ pasando a asumir esas funciones la Iglesia Cristiana.

En España, durante la Edad Media, esa labor hospitalaria se llevó a cabo por organizaciones monásticas, hermandades y entes diocesanos. Los obispados no sólo ejercían una notable influencia doctrinal sobre sus feligreses, sino que eran responsables de importantes labores asistenciales: limosnas, hospitales y otros servicios que ofrecían a la ciudad. En este sentido su poder era, sin duda, hegemónico respecto a los ayuntamientos de las localidades adscritas a la diócesis, pues la situación económica de los concejos locales, sometidos a numerosas contribuciones al Rey para costear las campañas militares, les impedían realizar esa labor humanitaria. Esta situación desembocaba con frecuencia en que el monarca pidiera auxilio económico a los obispos para aspectos urgentes relacionados con las poblaciones. Estos requerimientos de ayuda a los diferentes obispados no les sorprendían; de hecho era el Rey quien los nombraba, por ser personas de la nobleza de su confianza. ${ }^{4}$

Durante la Era Moderna, con el aumento poblacional, se multiplicaron las necesidades a cubrir por la beneficencia, situación que explica nuevas formas de asistencia social: las Casas de Misericordia, Albergues para pobres y Hospicios. ${ }^{5}$

${ }^{1}$ Entre las numerosas referencias, podemos señalar las del Evangelio de Mateo (capítulos V, VI y XXV), de Lucas y San Juan (capítulo XIII); Hechos de los Apóstoles (capítulos IV y V); y Corintios (capítulos VIII y IX).

${ }^{2}$ Arroyave, M. G.,e Isaza, P. 1989. «Una perspectiva histórica del hospital», Educación Médica y Salud 23/2: 182-191.

${ }^{3}$ La tradición mitológica señala a Esculapio como fundador de estos hospitales en el siglo IV a.C. (Labbé, M. et al,. 2008. «Símbolo de la medicina. Origen y evolución. El bastón de Esculapio versus Caduceo de Hermes. ¿Símbolos iguales o muy distintos?», Revista Chilena de Psiquiatría y Neurología de la infancia y adolescencia, 19/1: 60-66).

${ }^{4}$ Domínguez Ortiz, A. 1992. «La Sevilla del siglo XVII» en Historia de Sevilla, Morales Padrón, F. (dir.), tomo V. Universidad de Sevilla, 1992. El autor señala que «la carencia de un sistema sanitario público era suplida mediante limosnas episcopales y clericales, monasterios, conventos, cofradías y otras instituciones similares realizaron esta función social».

${ }^{5}$ Jiménez Salas, M. 1958. Historia de la asistencia social en España en la Era Moderna, Imprenta Viuda de Galo Sáez, Madrid. 
Son clásicos algunos autores que tratan la cuestión en el siglo XVI, tales como Vives, ${ }^{6}$ Domingo de Soto $^{7}$ y Villavicencio. ${ }^{8}$

Durante ese siglo Carlos I y Felipe II emiten sucesivas normas que intentan mejorar el funcionamiento de los hospitales del reino: por un lado, ordenan que se reduzcan en número, aglutinándose, para ser más eficientes; y por otro lado, sugieren que se creen nuevos hospitales, pero de un mayor tamaño.

La literatura ha estudiado este tipo de entidades hospitalarias centrándose en la labor asistencial realizada. Sin embargo, no abundan los estudios que aborden el funcionamiento de los mismos desde una perspectiva económica partiendo de los registros contables conservados de los mismos. Sí hay que destacar los estudios realizados sobre los hospitales de Santa Cruz de Toledo, de San Julián de Albacete y de Santa María de Plasencia. El objetivo del presente trabajo es analizar el funcionamiento de una entidad distinta de las anteriores por estar dedicada a dar alimento a los pobres, el Hospital de Santa Marta de Sevilla, a través de la información recogida en sus registros contables y, también como aspecto novedoso, analizando su labor a lo largo de cuatro siglos. ${ }^{?}$

\section{Sevilla y los hospitales: el Hospital de Santa Marta}

Los hospitales en Sevilla, como en el resto de España, empezaron a florecer tras la reconquista. Al principio eran pequeños de capacidad, y atendían indistintamente enfermos, viejos, pobres, niños abandonados y transeúntes pobres; es decir, eran hospitales, asilos y alberguerías. Ya en el siglo XIII se documentan más de 10 hospitales en la ciudad. ${ }^{10}$

${ }^{6}$ Vives, J.L. 1525. Del socorro de los pobres. Edición Biblioteca de Autores Españoles.

${ }^{7}$ Soto, D. de 1545.Deliberación en la causa de los pobres.

${ }^{8}$ Villavicencio, L. 1564. De oeconomia sacra circa pauperum curam. Amberes.

${ }^{9}$ Los estudios mencionados han sido realizados por Jiménez Montañés, M.A. y Villaluenga de Gracia, S. 2000. «Instituciones de acogida en Toledo en el siglo XVI, el Hospital de Santa Cruz: Aspectos contables». Ensayos sobre normalización, globalización e historia de la contabilidad. Universidad de Castilla La Mancha; López Pérez, M.M. y Pérez Morote, R. 2007. «El sistema de información contable del Hospital San Julián de Albacete entre 1838 y 1859. De Computis Revista Española de Historia de la Contabilidad. 6, 55-117; y Garzón Marín, P. y Donoso Anes, R. 2001. «El hospital de Santa María de Plasencia: características del método contable utilizado (1789-1839)». Comunicación presentada al XI Congreso de AECA, Madrid.

${ }^{10}$ Hospital de San Fernando, de San Clemente, de San Miguel, de San Lázaro, de Nuestra Señora de la Asunción, de Nuestra Señora de Rocamador, de San Mateo y otros de gremios parroquiales (Sánchez Herrero, J. 1992. «El clero de Sevilla en la época del barroco», en C. Ros (ed.), Historia de la Iglesia de Sevilla, 240-275. Sevilla: Editorial Castillejo). 
Durante los siglos XIV y XV el número de hospitales, la mayoría de ellos de pequeña dimensión, habría aumentado hasta el centenar. ${ }^{11}$ Entre los de nueva fundación podemos destacar el Hospital de la Misericordia, también denominado de San Cosme y San Damián, y vulgarmente conocido como «el de las Bubas». Estas entidades asistenciales se especializaron, ya fuera en la alimentación de los pobres, en la atención a «enfermos de calenturas», enfermos contagiosos, niños abandonados... muchas veces al amparo de cofradías nacidas de los gremios sevillanos. ${ }^{12}$

En el siglo XVI, como ya se ha comentado, la Corona intentó agrupar los hospitales para mejorar su eficacia. En Sevilla, cumpliendo esas normas, se fusionaron los entes hospitalarios en dos en el año 1587: el Hospital del Amor de Dios, y el Hospital del Espíritu Santo. Sin embargo, en la práctica, se sabe que otros muchos siguieron existiendo, incluso apareciendo otros nuevos. ${ }^{13}$

\section{El hospital de Santa Marta.}

Desde la Edad Media, en la diócesis de Sevilla, como en otras diócesis, ${ }^{14}$ debido a la presencia casi continuada de los obispos en la corte real, buena parte de las funciones diocesanas estaban delegadas en su Cabildo Catedral.

Una parte importante del presupuesto de la institución catedralicia estaba dirigida a una serie de patronatos, que eran fundaciones y obras pías, que se

\footnotetext{
${ }^{11}$ Ortiz de Zúniga, D. 1677. Annales Eclesiásticos y Seculares de la muy Noble y muy Leal Ciudad de Sevilla, Metrópoli de Andalucía, de la que contiene sus más principales memorias desde el año de 1246 hasta el año de 1671, Madrid: Imprenta Real.

${ }^{12}$ Sánchez Herrero, J. 1992. Ya a mediados del siglo xv había varias «cofradías hospitalarias» en la ciudad: las cofradías de los Santos Angeles, de San Bernabé, de San Bernardo, de los Inocentes, Santa María de la Concepción y de San Vicente.

${ }^{13}$ Collantes de Terán, F. 1886. Los establecimientos de caridad de Sevilla que se consideran como particulares: apuntes y memorias para su historia. Sevilla.

${ }^{14}$ Los obispos eran personas de gran influencia que solían estar siempre cerca de la corte real. Esto, agregado al hecho de que era frecuente que una misma persona fuera obispo de más de una diócesis, provocaba que muchas de las funciones de gobierno, tanto en materia espiritual como material, estuvieran delegadas en el Cabildo Catedral, en la persona de su deán. Esta cierta autonomía del Cabildo Catedralicio respecto al Arzobispo tiene su origen en las constituciones de don Remondo, que siguiendo las tendencias del resto de diócesis españolas del siglo XII, separaron las denominadas Mesa Episcopal o Arzobispal de la Mesa Capitular con atribuciones y fuentes de ingresos bien distinguidas (Lop Otín, M.J. 1996. «El siglo XII en la historia del Cabildo Catedral de Toledo», incluido en las actas del Congreso Internacional Conmemorativo del VIII Centenario de la Batalla de Alarcos, Universidad de Castilla-La Mancha, p. 479). En el caso sevillano, además se le otorgó al Cabildo la administración de los diezmos de la diócesis. Por tanto en ese largo período este órgano aglutinaba funciones de dirección tanto de la Catedral como templo, como del Arzobispado en su totalidad (Hernández Borreguero, J.J. 2010. La Catedral de Sevilla: economía y esplendor (siglos XVI y XVII), Sevilla: Ayuntamiento de Sevilla).
} 
instituían para dotar cultos (en determinadas fiestas, funciones litúrgicas, aniversarios de difuntos), y destinar fondos a instituciones benéficas tales como asilos, hospitales, dotes matrimoniales para doncellas, becas para estudiantes... ${ }^{15}$.

Estos patronatos catedralicios llegaron a ser muy numerosos, ${ }^{16}$ aunque muchos de ellos apenas consistían en dotaciones económicas que permitían mantener económicamente a un sacerdote para que realizara una serie de cultos por el alma de algún fallecido. Por el volumen económico destacan tres patronatos dependientes del Cabildo: ${ }^{17}$ el Hospital de Santa Marta, la Capilla de Scalas y el Colegio de San Isidoro.

El Hospital de Santa Marta se crea en 1404, con los bienes donados por un arcediano del Cabildo, Ferrán Martínez ${ }^{18}$ quien señala en su testamento «que para siempre sea hospital en que se reciban los pobres de Jesucristo». ${ }^{19}$ Durante siglos este hospital, situado donde ahora se encuentra el Convento de la Encarnación (edificio localizado entre la plaza Virgen de los Reyes y la Plaza del Triunfo), siguió cumpliendo la función encomendada por su fundador: dar alimento diariamente a los pobres, concretamente a unas 45-50 personas.

En este trabajo estudiamos a fondo el funcionamiento del Hospital de Santa Marta a partir de la realidad económica subyacente en los registros contables conservados correspondientes a los siglos XV a XVIII.

En el archivo de la Catedral se conservan más de 240 libros sobre la gestión de este hospital. La serie de los libros de cargo y descargo está prácticamente completa desde 1476 a 1824 , lo que ha facilitado nuestro trabajo.

Como veremos en esos libros de cuentas, todo hace indicar que los gestores del Cabildo (ya fueran los mayordomos durante los siglos XVI y parte del XVI, o ya fueran los contadores mayores desde la creación de sus cargos) exigieron que los administradores del hospital registraran sus ingresos y gastos mediante planes de cuentas, fijos, en los que había que encuadrarlos según su naturaleza,

\footnotetext{
${ }^{15}$ Rubio Merino, P. y González Ferrín, M.I. 1998. Inventario general del Archivo de la Santa, Metropolitana y Patriarcal Iglesia Catedral de Sevilla, Sevilla: Fundación Areces.

${ }^{16}$ El Archivo de la Catedral de Sevilla (ACS) conserva unos 1.000 libros de cuentas relacionados con este tipo de instituciones, con entidad jurídica propia e independencia económica, pero que eran tuteladas y sus cuentas revisadas por el Cabildo Catedral.

${ }^{17}$ Hernández Borreguero, J.J.

${ }^{18}$ Este arcediano fue famoso en su época tanto por su riqueza, poder y saber hacer dentro del Cabildo, como por las importantes donaciones que realizó al mismo. Sin embargo, también su fama procede de sus predicaciones contra el judaísmo, que incitaron al asalto al barrio de la judería de Sevilla, en el que murieron cientos de judíos. Por ello, estuvo preso durante algunos años (Montes Romero, I. 1998. Propiedad y explotación de la tierra de la Baja Edad Media. Sevilla: Editorial FOCUS).

${ }^{19}$ Rubio Merino, P. 1987. Inventario general del Archivo de la Santa, Metropolitana y Patriarcal Iglesia Catedral de Sevilla. Sevilla: Fundación Ramón Areces.
} 
siempre clasificados en el mismo orden. Su implantación, según nuestra opinión, obedece a tres causas: 1) la variedad y cantidad de partidas a controlar era muy reducida, favoreciendo la estructuración de la información económica 2) implementar y simplificar la revisión de la gestión económica a realizar por parte de la Contaduría Mayor y 3) facilitar la tarea de los administradores (que no sólo ejercían de contadores, sino de gestores del patronato), cuyo nivel de conocimientos de contabilidad eran muy reducidos (como puede deducirse tanto en la caligrafía utilizada, como en las explicaciones de los hechos contables).

Antes de analizar los libros dedicados a los gastos e ingresos anuales del hospital, haremos un comentario sobre 'los Protocolos', libros inventarios de las propiedades de la entidad.

\section{LOS INGRESOS MONETARIOS DEL HOSPITAL.}

Los ingresos asignados al Hospital estaban perfectamente delimitados, pues esta institución asistencial tenía una serie de bienes patrimoniales que le otorgaban suficientes réditos como para afrontar su actividad caritativa anual. Desde pocos años después de su Fundación, el Hospital de Santa Marta disfrutó de 54 propiedades inmobiliarias, casas, en su inmensa mayoría, además de algunos donadíos y tierras de cultivo.

Periódicamente el Hospital inventariaba esas propiedades en unos libros denominados Protocolos, siguiendo la tradicional política de conservación de sus documentos de las entidades eclesiásticas..$^{20}$ Estos libros, tras un índice, dedican una doble página a cada uno los bienes propiedad del Hospital, en la que apenas se menciona la ubicación geográfica del bien, pero se registran los sucesivos contratos de arrendamiento. Así, por ejemplo, en la página 38 del libro II de Protocolos, encontramos la anotación correspondiente a una casa de la calle Francos. Este registro comienza anotando el contrato de arrendamiento realizado en 1581, de por vida, ante escribano público, a favor de Pedro de Viola y su mujer, Sebastiana Ruiz, por 28.000 maravedíes (mrs). En 1608, se vuelve a arrendar tras la muerte de ambos, por el mismo importe a otra persona. Este arrendatario hizo dejación y devolvió en 1628 la casa, que se volvió a arrendar por $28.012 \mathrm{mrs}$, con obligación de hacer reparaciones.

\footnotetext{
${ }^{20}$ Aunque desde principios de la Edad Media la Iglesia puso un especial celo en el control y archivo de su documentación económica, esa tradición fue elevada a norma durante el Concilio de Trento, y fue desarrollada por el papa San Pío V, siguiendo los métodos que impuso en su diócesis San Carlos Borromeo, obispo de Milán, en cuanto a conservación e inventario de los archivos eclesiásticos. El Cabildo Catedral de Sevilla siguió escrupulosamente esta prerrogativa, por lo que no puede extrañarnos que hoy en día su archivo acumule más de 9.000 libros de cuentas para los siglos XV a XIX.
} 
Prácticamente todos estos inmuebles estaban permanentemente en alquiler, permitiendo al Hospital obtener unos importantes ingresos, como ya veremos más adelante. La mayoría de estos contratos de alquiler llevaban, una parte de la renta valorada en gallinas, una especie de cláusula de revalorización para actualizar parcialmente el ingreso en función de la subida del precio de un producto básico en la cesta de la compra. En la siguiente tabla, presentamos los datos del total de ingresos en concepto de arrendamiento obtenidos por el Hospital durante varios años correspondientes a los siglos XV a XVII, en los que puede comprobarse la parte de los ingresos que provenía de la liquidación de las gallinas recibidas:

\begin{tabular}{|l|r|r|r|r|r|}
\hline Concepto/Año & \multicolumn{1}{|c|}{$\mathbf{1 4 8 9}$} & \multicolumn{1}{c|}{$\mathbf{1 4 9 3}$} & $\mathbf{1 5 6 9}$ & \multicolumn{1}{c|}{$\mathbf{1 6 4 4}$} & \multicolumn{1}{c|}{$\mathbf{1 6 4 5}$} \\
\hline Posesiones & 47.093 & 62.104 & 587.528 & 1.203 .609 & 1.219 .968 \\
\hline Mrs gallinas & 1.930 & 3.240 & 167.809 & $417.027^{21}$ & 417.248 \\
\hline Total ingresos & 49.023 & 65.344 & 755.337 & 3.274 .432 & 1.637 .216 \\
\hline
\end{tabular}

Así, en el año 1644, el total de ingresos de la institución por estos conceptos ascendían a 1.203 .609 maravedíes y 2.809 gallinas. Estas gallinas proporcionaron 417.027 maravedíes $^{22}$ adicionales a la institución en ese año

Otros ingresos de naturaleza similar provenían de los denominados tributos, unas 14 partidas, muchos de ellos relacionados con alguna venta ${ }^{23}$ esporádica de un inmueble en el que el Hospital retenía el usufructo, por lo que percibía una determinada cantidad de manera perpetua. ${ }^{24}$ Ejemplos de esos casos son los tributos relacionados con de la Hacienda de Palomares, o la casa de Francisco de Vega. También aparecen tributos personales (a pagar por los herederos de Cristóbal Gallardo, o por Doña María de Villa) y los que pagaban algunas ins-

${ }^{21}$ Los 417.027 maravedíes provenían de las 2767 gallinas que se cobraron en moneda. En el libro de gastos del Hospital de ese año se especifica que a la mayoría de los arrendatarios se le valoraron las gallinas a 9 reales el par de gallinas (es decir, a 153 maravedíes la gallina). Solo dos arrendatarios que tenían que pagar 186 gallinas pudieron liquidarlas a 7 reales el par (a 119 maravedíes la unidad). Además otras 42 gallinas aparecen cobradas «en pluma» (es decir, se recibía el animal en cobro). Como es lógico pensar cobrando la mayor parte en metálico se evitaba el farragoso proceso de almacenamiento, mantenimiento y posterior venta de esos animales.

${ }^{22}$ En el libro de gastos del Hospital de ese año se especifica que a la mayoría de los arrendatarios se le valoraron las gallinas a 9 reales el par de gallinas (es decir, a 153 maravedíes la gallina). Solo dos arrendatarios que tenían que pagar 186 gallinas pudieron liquidarlas a 7 reales el par (a 119 maravedíes la unidad).

${ }^{23}$ La escasez de venta de posesiones era debida a la obligación legal (denominada vínculo) de mantenerla por la entidad receptora de la donación.

${ }^{24}$ Gregorio de Tejada, M. 1993. Vocabulario básico de la Historia de la Iglesia. Madrid: Editorial Crítica. 
tituciones (el Cabildo Catedral, la Fábrica, el Convento de San Benito de la Calzada, el Convento de la Encarnación, o el Hospital del Espíritu Santo).

Todos estos «tributos» proporcionaron ingresos sólo durante los siglos XVI y XVII (no hemos encontrado ninguna partida asimilable a esta categoría en los libros consultados de los siglos xV y XVIII). Como puede observarse en la siguiente tabla esos ingresos eran los más irregulares en importe:

\begin{tabular}{|l|r|r|r|r|}
\hline Concepto/Año & $\mathbf{1 5 6 9}$ & $\mathbf{1 5 7 0}$ & $\mathbf{1 6 4 4}$ & \multicolumn{1}{c|}{$\mathbf{1 6 4 5}$} \\
\hline Mrs tributos & 31939 & 17530 & 454662 & 227103 \\
\hline Total ingresos & 1062026 & 1179813 & 2978514 & 2742170 \\
\hline$\%$ Tributos/Total & $3,00 \%$ & $1,48 \%$ & $15,26 \%$ & $8,28 \%$ \\
\hline
\end{tabular}

El resto de ingresos tenía una menor cuantía, y están relacionados con aportaciones de la institución catedralicia, ya fuera directamente por medio del Cabildo o de la Fábrica de la misma. Suponemos que eran dotaciones que se concedían anualmente y que, por ejemplo, en el año 1644 ascendieron a 225.700 maravedíes.

El último concepto frecuente estaba relacionado con el superávit de los años anteriores, que se consideraba como un ingreso más. El año 1644 esa cifra alcanzaba los 903.216 maravedíes. Otros años, como 1625, ese importe estaba cercano a los 2 millones de maravedíes.

Dos aspectos más merecen nuestra atención desde un punto de vista contable: 1) Prácticamente todos los años las cuentas del Hospital arrojan un amplio superávit, que suponía entre un 20 y un $60 \%$ del total gastado, y que se denomina «alcance contra el administrador». Este superávit siempre es considerado como un ingreso más del ejercicio, por lo que se entiende que no existe un interés analítico en confrontar los ingresos y gastos devengados en el año en cuestión. 2) En algunos años aparecen ingresos que se clasifican como incobrables para el administrador (normalmente por problemas de solvencia de los arrendatarios). Esas partidas, denominadas «suspensiones y descuentos», curiosamente no se consideran como menores ingresos, sino como gastos del ejercicio.

LOS GASTOS MONETARIOS DEL HoSPITAL

Aunque los libros de registro de los gastos son muy similares durante los cuatro siglos analizados, hemos encontrado algunos matices que pasamos a explicar: 
a) En Los primeros libros conservados (años 1476 y 1477) es difícil recomponer el balance económico de la institución debido a la compleja grafía utilizada, a la simple ordenación cronológica de los gastos, y a la falta de un resumen final que confronte los gastos con los ingresos (en su mayoría no monetarios).

b) Esa situación cambia drásticamente en el siguiente libro catalogado (años 1488 a 1490): el administrador, Juan de Almodóvar, comienza a separar los gastos por categorías en páginas separadas. En el libro de los años 1492 a 1494 sigue la evolución de la administración contable, al introducirse el detalle diario de los principales gastos del hospital (como ya explicaremos más adelante): los de despensa.

c) Los libros estudiados del siglo XVI ya separan prácticamente tantos conceptos de gasto como los del siglo XVII y XVIII. Lo único que se mejoró en el siglo XVII respecto a éstos es que los de la anterior centuria totalizan los gastos por páginas y no por conceptos. Al final de esos libros aparecen todos esos subtotales sumados para totalizar los gastos del año. Este detalle sirve como ejemplo claro de que la principal función de estos libros no es servir para analizar la evolución de las distintas partidas de gasto, sino controlar la gestión de su administrador.

d) Como ya hemos adelantado, parece que los libros del siglo XVII suponen el máximo desarrollo contable, con las cuentas totalizadas por conceptos, y con un índice para encontrar con mayor facilidad las cuentas en él contenidas.

e) Los libros del siglo xvIII son casi idénticos a los anteriores. Solo destacar que pierden el índice inicial, y que separan los ingresos por arrendamientos temporales («de por tiempo») de los a largo plazo («de por vida»). También llama la atención el hecho de que los importes recogidos sufrieron numerosas correcciones posteriores (con una caligrafía y color de tinta distintos). No sabemos si fueron errores aritméticos o revisiones del Cabildo de los importes declarados.

Los administradores utilizaron una serie de clasificaciones y denominaciones que se mantuvieron estables durante más de 250 años, y recogen las sucesivas anotaciones que se realizan a lo largo del año. ${ }^{25}$ Nosotros hemos clasificado estos capítulos de gastos, a efecto de estudio, en tres bloques: los de carácter institucional, los relacionados con alimentos y los gastos generales.

\footnotetext{
${ }^{25}$ Hemos tenido noticias de que otros hospitales no realizaban estas ordenaciones de los gastos a partir de planes de cuentas, sino que cronológicamente van anotando los cargos y los descargos diarios, como sucede en el Hospital de Santa María, de Plasencia (Garzón Marín, P. y Donoso Anes, R. 2001).
} 
a) Gastos de carácter institucional: el primero que encajaría en este bloque son los que se acometían el día de la festividad de Santa Marta, que el hospital celebraba con gran solemnidad. Parte de esos gastos eran litúrgicos (pago al preste, sacristanes, acompañantes, colgaduras en el templo, flores...) y otra parte eran los gastos de alimentación de los acogidos en el hospital, que disfrutaban ese día de una comida especial (para lo que se solía comprar leche, apios, cestos de fruta, azúcar y especies).

Por otro lado, consideraremos como gastos institucionales las limosnas y misas ofrecidas por el hospital (pagadas por la institución y celebradas en algún templo). También se pagaban tributos a otras entidades (al convento de San Jerónimo, a la fábrica de San Salvador, a la fábrica de Santa Ana de Triana, al hospital de San Bernardo, al convento de San Pablo) y pequeñas cantidades a favor del Cabildo Catedral, su Fábrica y los capellanes del coro catedralicio. Esos tributos disminuyeron sensiblemente en el siglo XVIII.

b) Gastos relacionados con alimentos: el objetivo principal del hospital era proporcionar comida a una serie de pobres, identificados, que habían conseguido el derecho preferente a recibirla. De estos «beneficiarios» un alto porcentaje eran clérigos pobres; por ejemplo, a principios de 1644, recibían alimento 47 personas, 21 clérigos y 26 legos. Esa cifra de pobres alimentados es algo menor, por ejemplo, de la que aparece en 1625 (60 personas). El Hospital de Santa Marta concedía a cada una de estas personas el derecho a percibir una ración de alimentos. Esa porción de alimentos por persona solo fue detallada en los libros de cuentas del siglo XVII; a partir de éstos hemos podido averiguar que los trabajadores del hospital, además de su salario, recibían también una ración de alimentos; así, durante buena parte del siglo XVII recibían una ración: el despensero, dos criados, dos amas y el administrador jubilado; el administrador vigente recibía dos raciones. La magnitud que se controla contablemente no era el número de personas que recibían alimentos, sino el número de raciones entregadas; esta cifra oscilaba cada día entre 49 y 55. Al final de cada año se totalizaban las raciones ofrecidas, alcanzando la cifra de 18.921 en el año 1644; si tenemos en cuenta que se entregaban todos los días del año, obtendríamos una media de casi 52 raciones diarias. Comencemos a describir los gastos en productos alimenticios:

El gasto principal en alimentación fue en trigo, parte fundamental de la dieta diaria. Esto ocurrió durante los siglos XV a XVII, pues en el siglo XVIII no encontramos ningún gasto monetario relacionado. Durante algunos períodos la asignación de trigo que recibía el hospital era suficiente para conseguir el autoabastecimiento y para vender la parte restante. En la siguiente tabla vemos que los conceptos de gasto relacionados con el trigo y su transformación a pan eran variados: 


\begin{tabular}{|l|c|r|r|r|r|r|r|r|c|}
\hline $\begin{array}{l}\text { Gasto } \\
(\mathbf{m r s}) / \mathbf{a n ̃ o}\end{array}$ & $\mathbf{1 4 8 8}$ & $\mathbf{1 4 8 9}$ & $\mathbf{1 4 9 3}$ & $\mathbf{1 5 6 9}$ & $\mathbf{1 5 7 0}$ & $\mathbf{1 6 4 4}$ & $\mathbf{1 6 4 5}$ & $\mathbf{1 7 2 0}$ & $\mathbf{1 7 2 1}$ \\
\hline $\begin{array}{l}\text { Molino y } \\
\text { horno }\end{array}$ & 4572 & 4572 & 6469 & 0 & 0 & 0 & 0 & 0 & 0 \\
\hline $\begin{array}{l}\text { Trigo } \\
\text { comprado }\end{array}$ & 0 & 0 & 0 & 84346 & 144668 & 613156 & 339736 & 0 & 0 \\
\hline $\begin{array}{l}\text { Acarreto } \\
\text { pan }\end{array}$ & 2679 & 2530 & 0 & 0 & 0 & 0 & 0 & 0 & 0 \\
\hline
\end{tabular}

Como ejemplo de detalle de la tabla anterior señalaremos que a lo largo del año 1644 el administrador compró 7 partidas de grano de trigo, hasta totalizar 800 fanegas, que costaron 18.034 reales (613.156 maravedíes). Los precios por fanega de esas partidas oscilaron entre los 19 y 32 reales, demostrando las grandes oscilaciones en precio que sufría el trigo a lo largo de un año.

El segundo gasto en importancia era en vino, que solía adquirir en Villanueva del Ariscal. Así en el año 1644 se compraron, de una sola vez, 1.438 arrobas de vino, a 3 reales cada arroba. A ese gasto hubo que sumarle los impuestos (millones y testimonios) y el porte, hasta totalizar 359.671 maravedíes.

A continuación encontramos el gasto en leña, necesario para el funcionamiento de las cocinas. Este concepto no se reconoce como tal en el siglo xv, pero sí durante el resto de períodos. El gasto era bastante estable, así en el año 1644 el administrador del hospital hizo varias compras de leña, hasta totalizar 504 quintales de leña, que le costaron 63.563 maravedíes.

Otros productos esenciales en la cocina eran el aceite, tocino, garbanzos, castañas y arroz, cuyas compran en 1644 fueron las siguientes: se adquirieron en febrero 60 arrobas de aceite a 10 reales y medio la arroba; considerando el impuesto de millones, los gastos de acarreto y medidores, el coste final de ese aceite ascendió a 29.427 maravedíes; fueron de tocino, por importe de 44.404 maravedíes; 15 fanegas de garbanzos, por valor de 13.362 maravedíes; 8 arrobas de castañas (3.672 maravedíes) y otras 6 arrobas de arroz (6.732 maravedíes). Otro apartado recoge las compras de queso y frutas (entre ellas algunas partidas de queso de Flandes); este apartado solo aparece durante el siglo Xvi. Todas estas partidas tienen en común que se refieren a alimentos almacenables durante un período razonable de tiempo, a diferencia de los siguientes.

El resto de gastos alimenticios se agrupan en el apartado de «semanerías y despensa», en los que se recogen los productos comprados en cantidades de pequeño coste económico (azafrán, comino, sal, afrecho, culantro, ${ }^{26}$ lentejas); también

\footnotetext{
${ }^{26}$ Según el Diccionario de Autoridades es sinónimo de cilantro, «especie de granillos redondos para sazonar los alimentos».
} 
aparecen numerosos alimentos perecederos (carnero, puerco, bacalao, sardinas, cazón, huevos, coles, nabos, perejil, cebolla); utensilios y productos de limpieza (jabón, lejía, compra de una capacha, velas, manteles); por último destacaremos el reparto entre los pobres «en nómina» del Hospital de cierto importe monetario el Jueves Santo, pues permanecía cerrado el hospital desde ese jueves hasta el Sábado Santo, para que pudieran pagar su alimentación durante esos días de fiesta.

Muchos de esos gastos se contabilizan por semanas (de domingo a sábado), para la cual se registran relaciones diarias de gastos ordinarios, sobre todo alimentos: carnes, puercos, azafrán, huevos, sal... Se hallan los totales semanales y después mensuales, hasta obtener el total anual. Como ya hemos señalado algunos alimentos y condimentos se controlaban en cuentas aparte, como el trigo, aceite y vinagre o tocino, y otros simplemente se reflejen en las relaciones diarias de gastos: carnes, huevos, sal...; además otros alimentos tales como garbanzos, cebollas y castañas se registran como extraordinarios.

Estos gastos de despensa eran los más numerosos, y representaban un importante porcentaje del total de los gastos del Hospital, como puede verse en la siguiente tabla:

\begin{tabular}{|l|c|c|c|c|c|c|c|c|}
\hline Gasto/año & $\mathbf{1 4 8 8}$ & $\mathbf{1 4 8 9}$ & $\mathbf{1 5 6 9}$ & $\mathbf{1 5 7 0}$ & $\mathbf{1 6 4 4}$ & $\mathbf{1 6 4 5}$ & $\mathbf{1 7 2 0}$ & $\mathbf{1 7 2 1}$ \\
\hline $\begin{array}{l}\text { Semanerías } \\
\text { y despensa }\end{array}$ & 37587 & 37405 & 210825 & 209105 & 646295 & 625839 & 388821 & 394425 \\
\hline $\begin{array}{l}\text { \% Total } \\
\text { gastos }\end{array}$ & $3,29 \%$ & $3,00 \%$ & $4,87 \%$ & $10,59 \%$ & $15,31 \%$ & $18,01 \%$ & $13,30 \%$ & $15,61 \%$ \\
\hline
\end{tabular}

c) Gastos generales: el resto de salidas económicas de la institución las hemos encuadrado en este apartado. Entre todos estos destaca el importe pagado en salarios al personal que trabajaba en el hospital. Los sueldos pagados eran exiguos, lo cual no nos sorprende si tenemos en cuenta que sus labores eran poco cualificadas y que los empleados comían gratis en el comedor. Como ejemplo de esas retribuciones traemos aquí las distribuidas por el Hospital de Santa Marta en el año 1644: el administrador (el licenciado Pablo Díaz), responsable de la gestión del hospital, apenas percibía 27.700 maravedíes anuales. El administrador jubilado, Sebastián Sobrino, percibe, suponemos que a modo de pensión (pues no sabemos si colaboraba en las tareas de la institución), 10.200 maravedíes. El despensero percibía 12.240 maravedíes. Cada una de las dos amas (Catalina de Jerez e Isabel de Mesa) cobraba 10.200 maravedíes anuales. Finalmente Martín Suárez, como contador mayor encargado de supervisar la parcela económica de la gestión del Hospital de Santa Marta, percibía 2.700 maravedíes anuales. En total, se pagaron en 1644 por conceptos salariales 
72.240 maravedíes, llamándonos la atención que los dos criados antes mencionados no percibieran salario, y tan solo recibieran una ración diaria de alimentos. Como decíamos estas retribuciones no eran muy generosas si tenemos en cuenta que el notario de la Fábrica de la Catedral percibió ese mismo año 130.900 maravedíes, el oficial mayor de la misma ganaba 62.500 maravedíes, y el resto de oficiales de la Fábrica cobraban entre 26.772 y 52.311 maravedíes.

Otros años se pagan salarios a un contador, un letrado, y un procurador mayor; $\mathrm{y}$ otros cargos de menor entidad: dos escribanos del vino y «al que visita los vinos». En los «salarios de esta casa que paga a los servidores de ella» se pagan a 6 personas. ${ }^{27}$

El nombre genérico de gastos generales era utilizado por el administrador para agrupar gastos administrativos (compra de papel y pliegos sellados, mandaderos que traían papeles y pagaban tasas), gastos de mantenimiento («aderezo de una cerradura») y pequeñas compras de utensilios (cucharas grandes de hierro o escobas). Algunos de estos conceptos podrían considerarse como gastos extraordinarios, sin embargo, en algunos períodos se creó ese otro apartado, dando lugar a unos importes altamente inestables, como puede comprobarse en la siguiente tabla:

\begin{tabular}{|l|r|r|r|r|r|r|r|r|r|}
\hline Gasto/año & $\mathbf{1 4 8 8}$ & $\mathbf{1 4 8 9}$ & $\mathbf{1 4 9 3}$ & $\mathbf{1 5 6 9}$ & $\mathbf{1 5 7 0}$ & $\mathbf{1 6 4 4}$ & $\mathbf{1 6 4 5}$ & $\mathbf{1 7 2 0}$ & $\mathbf{1 7 2 1}$ \\
\hline $\begin{array}{l}\text { Otros gastos } \\
\text { generales }\end{array}$ & 1.944 & 20.346 & 2.896 & 81.682 & 5.219 & 22.593 & 25.728 & 9.638 & 15.848 \\
\hline Extraordinarios & 0 & 0 & 575 & 18.001 & 20.216 & 0 & 0 & 0 & 0 \\
\hline
\end{tabular}

Otro capítulo de gastos eran los de «pleitos y libramientos», por los que el hospital aporta pequeñas cantidades al Cabildo como prorrateo de gastos que tenía que acometer de forma corporativa (denominados «libranzas de contaduría»); y dotaciones de provisiones de fondos para varios pleitos.

También se le dedicó un apartado para los gastos que afrontó el Hospital para realizar pequeñas reformas dentro de sus dependencias. Así en el año 1644 se detallan los materiales adquiridos (compra de una tabla, un cajón, dos docenas de clavos grandes, cal y yeso) y las personas que realizaron la obra (empedradores, un carpintero y un pintor), hasta totalizar 2.112 maravedíes.

Finalmente haremos mención a las gallinas que se pagaron «en pluma» a diversas personalidades. Éstas, aunque se mencionan en el apartado de los gastos, no

\footnotetext{
${ }^{27}$ Esas seis personas debían firmar 'el recibí' en el mismo libro, pero en la mayoría de los casos firmaba el escribano «por no saber firmar»
} 
aparecen valoradas monetariamente en el resumen total de gastos, pues ya habían sido sustraídas de los ingresos. Los importes pagados en especie corresponden a parte de la retribución recibida (por ejemplo en el año 1644) por el visitador del año (12 gallinas), un contador mayor (6), el procurador mayor (6), un letrado (6), el escribano del juzgado (4), los escribanos del puente (4), el comprador de vinos (2) y el escribano del cajón de los millones (2), hasta totalizar 42 gallinas.

A continuación de las páginas dedicadas a los gastos, cada libro de cuentas anual terminaba con el cierre y resumen de cuentas, que solía denominarse fenecimiento de la cuenta. Consistía en recopilar todos los importes de ingresos y gastos, calcular el superávit a cuenta nueva, y dejar constancia del alcance contra el administrador, quien debía de responder sobre el saldo final sobrante del ejercicio (véase en el anexo I el resumen final de las cuentas del año 1625).

En el anexo II resumimos todos los gastos e ingresos del Hospital durante una serie de años de los siglos XV a XVIII. Como puede comprobarse hemos reconstruido la cuenta resultados de la institución cada 70-75 años, y hemos recopilado las cifras de dos años seguidos, para así relativizar el hecho de que alguna partida tuviera un importe poco frecuente.

\section{INGRESOS Y GASTOS EN PAN}

Como hemos mencionado, dentro de los ingresos asignados al Hospital de Santa Marta se encuentran, aparte de los valorados en unidades monetarias y gallinas, los de grano de trigo. Durante los siglos XV y xVI el Hospital se autoabastecía con el trigo recibido, e incluso vendía una parte importante del grano disponible; en los siglos XVII y XVIII casi todo el trigo se compraba. Ambos importes (el trigo recibido y comprado) se totalizaban en el cargo de pan, como puede comprobarse en la siguiente tabla, expresada en unidades de peso (fanegas y almudes, la doceava parte de una fanega):

\begin{tabular}{|l|c|c|c|}
\hline Concepto/año & $\mathbf{1 4 8 9}$ & $\mathbf{1 5 6 9}$ & $\mathbf{1 6 4 4}$ \\
\hline Cargo de $\operatorname{pan}^{28}$ & $347 \mathrm{f} 6 \mathrm{al}^{28}$ & $479 \mathrm{f} 6 \mathrm{al}$ & $839 \mathrm{f} 4 \mathrm{al}$ \\
\hline
\end{tabular}

${ }^{28}$ El trigo se medía en unidades de peso: un cahiz (c) equivalía a 12 fanegas (f), y 12 almudes (al) hacían una fanega. Una fanega de trigo ocuparía un recipiente de 55,5 litros, y esta cantidad puede estimarse en 44,4 kilogramos de peso (Ladero Quesada, M.A. y González Jiménez, M. 1978. Diezmo eclesiástico y producción de cereales en el reino de Sevilla (1408-1503). Universidad de Sevilla, Departamento de Historia Medieval).

${ }^{29}$ En el siglo xv, además de recibir esa cantidad de trigo, recibió la mitad de ese importe en cebada (14 cahices y 4 fanegas), totalizando más de 520 fanegas de grano. 
La data del pan se corresponde con el trigo consumido, agrupable en tres conceptos, que explicamos tomando como ejemplo nuevamente el año 1644: por un lado se menciona que se emplearon 8 fanegas y 11 almudes de trigo «en la cocina para la mostaza y especies»; también se necesitaba una pequeña cantidad de grano para molerla «para los fritos de los días de pescado del año» ( 2 fanegas). El consumo principal se originaba por la transformación en pan del grano. Como el hospital no disponía de horno, se lo entregaba a un panadero que, a cambio, daba 20 hogazas de pan por cada fanega (cantidad que se redujo en el período mayo-diciembre a 19 hogazas). Sin duda, la cantidad de hogazas recibidas era menor que las que se podían obtener de una fanega de trigo, pero la retribución al panadero iba implícita en ese trato. Periódicamente se entrega al panadero un importe de fanegas para ir cuadrando las hogazas que se recibían con las que se entregan dentro del hospital (a razón de media hogaza diaria por ración). Al final del año, fruto de la transformación a pan, se consumieron 490 fanegas y 4 almudes de trigo.

Como puede comprobarse en el siguiente cuadro, el volumen de trigo empleado en el año 1644 apenas superó la mitad del recibido (incluyendo el comprado). Teniendo en cuenta que el consumo anual de trigo era muy similar podemos suponer que el administrador del hospital decidió comprar un volumen de grano tan grande para evitar inesperadas subidas del precio del grano a principios de año:

\begin{tabular}{|l|c|}
\hline CUENTA DEL PAN & Importe \\
\hline Alcance administrador anterior & $24 \mathrm{f} 4 \mathrm{al}$ \\
\hline Tributo en Carmona cobrado & $15 \mathrm{f}$ \\
\hline Trigo comprado & $800 \mathrm{f}$ \\
\hline TOTAL CARGOS & $839 \mathrm{f} 4 \mathrm{al}$ \\
\hline Pan gastado en raciones & $490 \mathrm{f} 4$ \\
\hline Gastado en la mostaza y fritos & $10 \mathrm{f} 11 \mathrm{al}$ \\
\hline TOTAL DATAS & $501 \mathrm{f} 3 \mathrm{al}$ \\
\hline ALCANCE 1644 & $338 \mathrm{f} 1 \mathrm{al}$ \\
\hline
\end{tabular}

\section{Conclusiones}

Como hemos podido comprobar a lo largo del trabajo, la institución hospitalaria cumplió con la función establecida por el fundador del Hospital de dar alimento a los pobres, labor perfectamente adecuada y promovida por la moral cristiana. 
Esa labor se realizaba de una manera organizada, coherente y eficaz, pues una serie de personas inmersas en la pobreza disfrutaban de una alimentación bastante digna y variada durante todos los días del año.

Además, la institución proporcionaba trabajo y comida a una serie de personas, con un alto grado de autonomía a la hora de decidir los alimentos a ofrecer diariamente. Como hemos visto en el Anexo II, la institución atendió durante siglos a una cifra similar de pobres, pues dispuso de ingresos suficientes para poder sufragar las necesidades alimenticias de estas personas, incluso en períodos de carestías, hambrunas o epidemias que encarecían los productos y, por tanto, aumentaban los gastos del Hospital.

El Cabildo Catedral ejercía la tutela de la institución, supervisando anualmente las cuentas para evitar el fraude o el mal uso de los fondos de su administrador. Esta labor se veía enormemente favorecida por el uso de sencillos planes de cuentas para clasificar los ingresos y gastos del Hospital, que se preservaron durante siglos.

Esa organización sencilla de los ingresos y gastos favoreció la labor de los administradores, que más que simples contadores ${ }^{30}$ profesionales, ejercían la dirección efectiva de todo lo relacionado con el Hospital. Esa reducción de la dificultad de contabilización no estaba reñida con el minucioso detalle de los precios y cantidades de productos comprados, aunque supusieran pequeños importes económicos.

Finalmente, queremos resaltar la labor de los administradores, quienes conocen al comenzar el año prácticamente el presupuesto exacto de ingresos, y se dedican a vigilar el gasto, para amoldarlo a las posibilidades presupuestarias y para equilibrarlo con la necesidad de cumplir los objetivos para los que se creó el patronato. ${ }^{31}$

${ }^{30}$ Martín Lamoroux, F. 1998. «Los criterios conceptuales y formales en la Historia de la Contabilidad», Ponencia al II Encuentro de trabajo sobre la Historia de la Contabilidad en España, Sevilla, señala que los administradores han de someterse a los procesos de rendición de cuentas impuestos por los destinatarios de la información; cuando éstos exigen que se lleven las cuentas mediante el método de cargo y data buscan que la rendición de cuentas sea un proceso simple.

${ }^{31}$ Lightbody, Margaret. 2000. «Storing and shielding: financial management behaviour in a church organization». Accounting, Auditing and Accountability Journal. 13/2: 165. Algunos autores han estudiado esta realidad precisamente en los hospitales. Harris, J. 1977. «The internal organization of hospitals: some economic indicators», The Bell Journal of Economics, 8: 467-82. 
BIBLIOGRAFÍA

Libros del archivo Catedral de Sevilla consultados:

- Protocolos I y II, libro de las posesiones del hospital de Santa Marta (2 libros) [1543-1645]. Sección Patronatos, ref. 32-33.

- Libros de los gastos ordinarios y extraordinarios del hospital de Santa Marta (10 libros) [1476-1721]. Sección Patronatos, ref. 38, 39, 40, 90, 91, 145, 162, 163, 234 y 235.

Arroyave, María de Gracia e Isaza, Pedro. 1989. «Una perspectiva histórica del hospital», Educación Médica y Salud 23/2: 182-191.

Collantes de Terán, Francisco. 1886. Los establecimientos de caridad de Sevilla que se consideran como particulares: apuntes y memorias para su historia. Sevilla.

Domínguez Ortiz, Antonio. 1992. «La Sevilla del siglo XVII» en Historia de Sevilla, Morales Padrón, F. (dir.). Universidad de Sevilla, 1992. El autor señala que «la carencia de un sistema sanitario público era suplida mediante limosnas episcopales y clericales, monasterios, conventos, cofradías y otras instituciones similares realizaron esta función social».

Garzón Marín, Pilar y Donoso Anes, Rafael. 2001. «El hospital de Santa María de Plasencia: características del método contable utilizado (1789-1839)». Comunicación presentada al XI Congreso de AECA, Madrid.

Gregorio de Tejada, Manuel. 1993. Vocabulario básico de la Historia de la Iglesia. Madrid: Editorial Crítica.

Harris, John. 1977. «The internal organization of hospitals: some economic indicators», The Bell Journal of Economics, 8: 467-82.

Hernández Borreguero, José Julián. 2010. La Catedral de Sevilla: economía y esplendor (siglos XVI y XVII), Sevilla: Ayuntamiento de Sevilla.

Jiménez Montañés, María Ángeles y Villaluenga de Gracia, Susana. 2000. «Instituciones de acogida en Toledo en el siglo xvı, el Hospital de Santa Cruz: Aspectos contables», Ensayos sobre normalización, globalización e historia de la contabilidad, págs. 343-370. Universidad de Castilla La Mancha.

Jiménez Salas, María. 1958. Historia de la asistencia social en España en la Era Moderna. Madrid: Imprenta Viuda de Galo Sáez.

Labbé, Mariana et al,. 2008. «Símbolo de la medicina. Origen y evolución. El bastón de Esculapio versus Caduceo de Hermes. ¿Símbolos iguales o muy distintos?», Revista Chilena de Psiquiatría y Neurología de la infancia y adolescencia, 19/1: 60-66.

Ladero Quesada, Miguel Ángel y González Jiménez, Manuel. 1978. Diezmo eclesiástico y producción de cereales en el reino de Sevilla (1408-1503). Universidad de Sevilla, Departamento de Historia Medieval

Lightbody, Margaret. 2000. «Storing and shielding: financial management behaviour in a church organization». Accounting, Auditing and Accountability Journal. 13/2: 156-170. 
Lop Otín, María José. 1996. «El siglo XII en la historia del Cabildo Catedral de Toledo», incluido en las actas del Congreso Internacional Conmemorativo del VIII Centenario de la Batalla de Alarcos, Universidad de Castilla-La Mancha: 471-494.

López Pérez, María del Mar y Pérez Morote, Rosario. 2007. «El sistema de información contable del Hospital San Julián de Albacete entre 1838 y 1859. De Computis Revista Española de Historia de la Contabilidad: 6, 55-117.

Martín Lamoroux, Fernando. 1998. «Los criterios conceptuales y formales en la Historia de la Contabilidad», Ponencia al II Encuentro de trabajo sobre la Historia de la Contabilidad en España, Sevilla.

Montes Romero, Isabel. 1998. Propiedad y explotación de la tierra de la Baja Edad Media. Sevilla: Editorial FOCUS

Ortiz de Zúniga, Diego. 1677. Annales Eclesiásticos y Seculares de la muy Noble y muy Leal Ciudad de Sevilla, Metrópoli de Andalucía, de la que contiene sus más principales memorias desde el año de 1246 hasta el año de 1671, Madrid: Imprenta Real.

Rubio Merino,Pedro. 1987. Inventario general del Archivo de la Santa, Metropolitana y Patriarcal Iglesia Catedral de Sevilla. Sevilla: Fundación Ramón Areces.

Rubio Merino, Pedro y González Ferrín, María Isabel. 1998. Inventario general del Archivo de la Santa, Metropolitana y Patriarcal Iglesia Catedral de Sevilla, Sevilla: Fundación Areces.

Sánchez Herrero, Juan. 1992. «El clero de Sevilla en la época del barroco», en C. Ros (ed.), Historia de la Iglesia de Sevilla: 240-275. Sevilla: Editorial Castillejo.

Soto, Domingo de.1545.Deliberación en la causa de los pobres.

Villavicencio, Lorenzo. 1564. De oeconomia sacra circa pauperum curam. Amberes.

Vives, Juan Luis. 1525. Del socorro de los pobres. Edición Biblioteca de Autores Españoles. 
Anexo I. Resumen cuentas Hospital Santa Marta, 1625

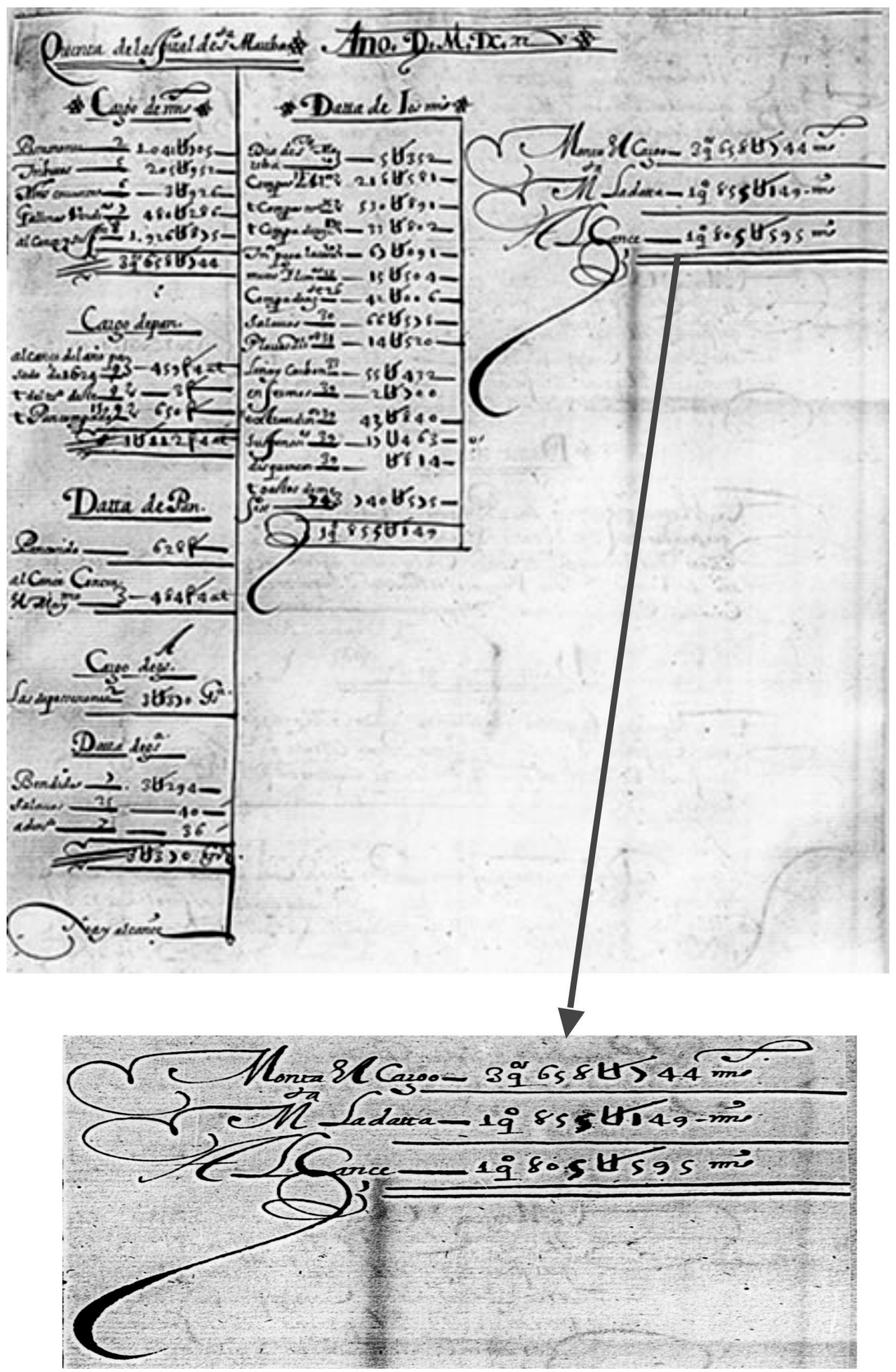

Hispania Sacra, LXVII 136, julio-diciembre 2015, 613-633 ISSN: 0018-215X, doi: 10.3989/hs.2015.018 


\begin{tabular}{|c|c|c|c|c|c|c|c|c|c|c|c|c|c|c|c|}
\hline$\underline{\mathbb{}}$ & 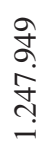 & $\begin{array}{l}\text { J } \\
\stackrel{9}{\circ} \\
\frac{\Omega}{1}\end{array}$ & 0 & 0 & 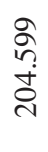 & 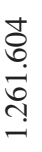 & 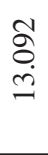 & $\begin{array}{l}\stackrel{\sim}{\infty} \\
\infty \\
\end{array}$ & 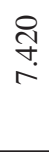 & 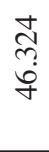 & 0 & 0 & 0 & $\begin{array}{l}\text { Oे } \\
\text { ò } \\
\infty \\
\text { I }\end{array}$ & $\begin{array}{l}\mathfrak{a} \\
\hat{b} \\
\stackrel{0}{0}\end{array}$ \\
\hline ్ㅗ & 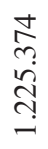 & 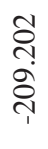 & 0 & 0 & 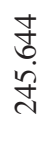 & 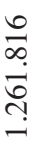 & 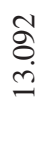 & $\frac{\text { ơ }}{n}$ & $\begin{array}{l}\infty \\
\stackrel{0}{0} \\
\dot{ \pm}\end{array}$ & $\begin{array}{l}\stackrel{+}{+} \\
\text { in } \\
\text { in }\end{array}$ & 0 & 0 & 0 & $\begin{array}{l}\stackrel{\infty}{+} \\
\stackrel{+}{\exists}\end{array}$ & $\begin{array}{l}\text { Pे } \\
\text { n’ } \\
\text { on }\end{array}$ \\
\hline$\underset{d}{\mathbb{E}}$ & 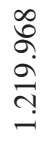 & 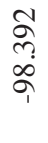 & 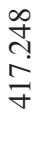 & $\begin{array}{l}\stackrel{0}{0} \\
\stackrel{ }{N}\end{array}$ & 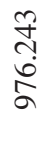 & 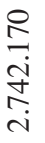 & $\begin{array}{l}\circ \\
\text { O̊ } \\
=\end{array}$ & $\begin{array}{l}\stackrel{\sim}{ } \\
\stackrel{n}{n}\end{array}$ & $\begin{array}{l}\stackrel{2}{\infty} \\
\infty \\
\infty\end{array}$ & $\begin{array}{l}\infty \\
\infty \\
\dot{J} \\
\Xi\end{array}$ & 0 & $\begin{array}{l}\stackrel{0}{r} \\
\hat{\sigma} \\
m\end{array}$ & 0 & 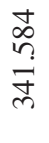 & 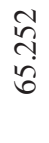 \\
\hline$\underset{U}{\mathbb{J}}$ & 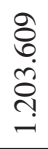 & & 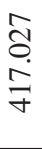 & 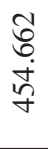 & 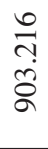 & 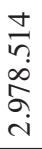 & $\begin{array}{l}\infty \\
\Xi\end{array}$ & 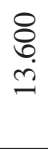 & $\begin{array}{l}\mathbb{J} \\
\infty \\
\infty \\
\infty\end{array}$ & 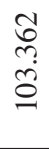 & 0 & $\frac{\bullet}{\frac{n}{n}}$ & 0 & $\begin{array}{l}\bar{\sigma} \\
0 \\
0 \\
m\end{array}$ & 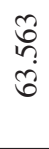 \\
\hline$\underset{n}{R}$ & $\frac{\infty}{=}$ & 0 & 0 & $\begin{array}{l}\text { nn } \\
\text { ṇ } \\
\end{array}$ & $\begin{array}{l}\sqrt{6} \\
\text { मे } \\
\text { ले }\end{array}$ & $\begin{array}{l}\frac{m}{\infty} \\
\stackrel{\infty}{2} \\
= \\
=\end{array}$ & $\underset{-}{\mathbb{Z}}$ & $\begin{array}{l}\stackrel{\oplus}{\oplus} \\
\stackrel{\oplus}{\sim}\end{array}$ & $\frac{\text { ণิ }}{\text { †े }}$ & $\begin{array}{l}\infty \\
\text { } \\
\text { గె } \\
\end{array}$ & 0 & $\begin{array}{l}\infty \\
0 \\
0 \\
\dot{J} \\
\dot{J}\end{array}$ & 0 & 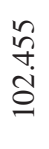 & 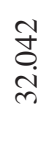 \\
\hline 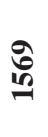 & 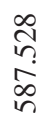 & 0 & $\begin{array}{l}\stackrel{8}{0} \\
\text { ஸे }\end{array}$ & $\frac{\text { ळे }}{\text { m }}$ & 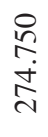 & 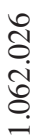 & ঔ̊ & $\begin{array}{l}\underset{J}{J} \\
\stackrel{J}{J}\end{array}$ & 0 & $\begin{array}{l}\overline{\text { }} \\
\stackrel{ \pm}{ \pm}\end{array}$ & 0 & 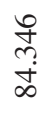 & 0 & $\begin{array}{l}0 \\
\text { ô } \\
\stackrel{0}{=}\end{array}$ & $\begin{array}{l}\vec{n} \\
\stackrel{\sim}{ה}\end{array}$ \\
\hline g̊ & 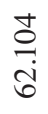 & 0 & 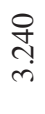 & 0 & 0 & $\begin{array}{l}\frac{7}{2} \\
n \\
n\end{array}$ & $\frac{0}{\infty}$ & 0 & $\begin{array}{l}\text { D̊ } \\
\text { గִ }\end{array}$ & $\begin{array}{l}0 \\
\stackrel{\sim}{\infty} \\
\infty\end{array}$ & 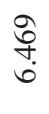 & 0 & 0 & 0 & 0 \\
\hline$\stackrel{\infty}{\stackrel{\infty}{+}}$ & $\begin{array}{l}\stackrel{2}{\delta} \\
\stackrel{\sigma}{\sigma}\end{array}$ & 0 & ڤి & 0 & $\begin{array}{l}\tilde{n} \\
\tilde{n} \\
\sim\end{array}$ & $\begin{array}{l}\frac{n}{\hat{a}} \\
\text { to }\end{array}$ & 0 & 0 & $\begin{array}{l}\stackrel{\overbrace{}}{N} \\
\stackrel{0}{0}\end{array}$ & $\begin{array}{l}\stackrel{\overbrace{}}{N} \\
\stackrel{0}{0}\end{array}$ & $\underset{i n}{\stackrel{N}{*}}$ & 0 & $\begin{array}{l}\text { n } \\
\text { nn } \\
\text { in }\end{array}$ & 0 & 0 \\
\hline$\stackrel{\infty}{\stackrel{\infty}{+}}$ & & & & & & a. & 0 & 0 & $\begin{array}{l}\stackrel{0}{0} \\
\text { i } \\
\text { In }\end{array}$ & $\begin{array}{l}\stackrel{0}{0} \\
\text { ¿ } \\
\end{array}$ & $\underset{\forall}{\stackrel{N}{r}}$ & 0 & $\begin{array}{l}\stackrel{a}{\sigma} \\
\stackrel{i}{1}\end{array}$ & 0 & 0 \\
\hline 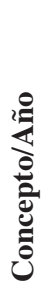 & 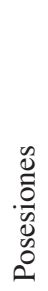 & 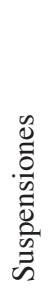 & 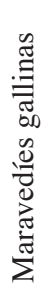 & 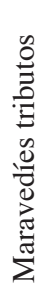 & 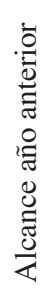 & 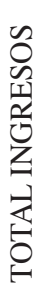 & 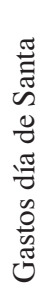 & 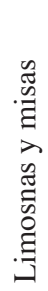 & 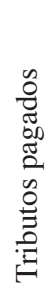 & 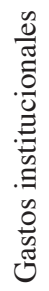 & 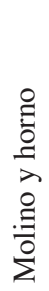 & 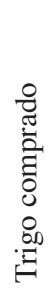 & 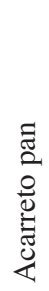 & 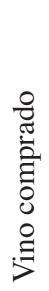 & 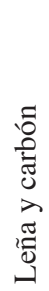 \\
\hline
\end{tabular}

Hispania Sacra, LXVII

136, julio-diciembre 2015, 613-633 ISSN: 0018-215X, doi: 10.3989/hs.2015.018 
EL HOSPITAL DE SANTA MARTA DE SEVILLA: ACTIVIDADES Y GESTIÓN...

\begin{tabular}{|c|c|c|c|c|c|c|c|c|c|c|c|c|c|c|}
\hline$\frac{\curvearrowright}{\stackrel{N}{\sim}}$ & 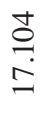 & $\begin{array}{l}\stackrel{0}{n} \\
\stackrel{\sim}{n} \\
\text { nn }\end{array}$ & 0 & 0 & \begin{tabular}{l}
$\stackrel{2}{\mathcal{J}}$ \\
\multirow{+}{*}{} \\
ले
\end{tabular} & $\frac{\tilde{n}}{\stackrel{n}{n}}$ & $\frac{\stackrel{8}{+}}{i}$ & 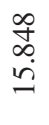 & 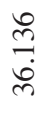 & $\begin{array}{l}0 \\
\stackrel{\sigma}{\alpha} \\
\stackrel{a}{=}\end{array}$ & 0 & $\begin{array}{l}\stackrel{Q}{+} \\
\stackrel{\oplus}{+} \\
\stackrel{\sim}{d}\end{array}$ & $\begin{array}{l}\stackrel{0}{=} \\
\stackrel{a}{\sigma}\end{array}$ & $\begin{array}{l}\infty \\
\stackrel{\infty}{+} \\
\stackrel{+}{+} \\
\text { ஸे }\end{array}$ \\
\hline 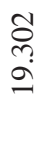 & 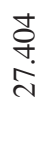 & $\begin{array}{l}\text { ọ } \\
\text { f. } \\
\text { on }\end{array}$ & 0 & 0 & $\begin{array}{l}\vec{N} \\
\infty \\
\infty \\
\infty \\
\infty\end{array}$ & $\frac{\hbar}{\hat{\sigma}}$ & $\frac{\stackrel{9}{r}}{i}$ & $\begin{array}{l}\infty \\
\text { లె. } \\
\stackrel{\sigma}{0}\end{array}$ & $\begin{array}{l}\tilde{N} \\
\stackrel{+}{ \pm}\end{array}$ & 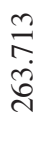 & 0 & 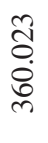 & $\begin{array}{l}\frac{N}{\text { Na }} \\
\text { ñ } \\
0\end{array}$ & 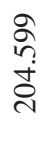 \\
\hline 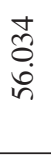 & $\begin{array}{l}\infty \\
\text { లి } \\
\stackrel{\sim}{े}\end{array}$ & $\begin{array}{l}\text { ळ̆ } \\
\text { ă }\end{array}$ & $\begin{array}{l}\stackrel{2}{~} \\
\infty\end{array}$ & 0 & $\begin{array}{l}\text { సે } \\
\text { ஸे } \\
\text { రુ }\end{array}$ & 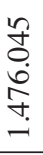 & 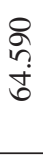 & 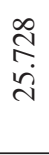 & $\begin{array}{l}\infty \\
\sim \\
\tilde{n} \\
\ddot{n}\end{array}$ & $\begin{array}{l}\stackrel{\infty}{\infty} \\
\text { ஸ̣ }\end{array}$ & 0 & $\begin{array}{l}\mathscr{0} \\
\stackrel{+}{+} \\
=\end{array}$ & 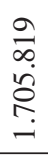 & 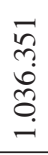 \\
\hline 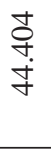 & $\begin{array}{l}\text { I. } \\
\text { ปे. }\end{array}$ & $\begin{array}{l}\text { ర్ } \\
\text { nִ } \\
\end{array}$ & 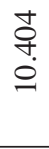 & 0 & 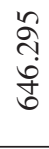 & 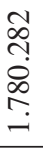 & 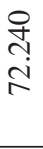 & 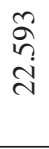 & $\begin{array}{l}\text { OO } \\
0 \\
0 \\
\vec{\sim}\end{array}$ & $\begin{array}{l}\cong \\
\Xi \\
\sim\end{array}$ & 0 & $\begin{array}{l}\hat{\sigma} \\
\infty \\
=\end{array}$ & 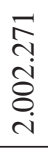 & 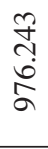 \\
\hline $\begin{array}{l}n \\
\infty \\
\infty \\
\hat{\sim}\end{array}$ & $\begin{array}{l}\text { बे } \\
\text { ¿े }\end{array}$ & 0 & 0 & $\begin{array}{l}\hat{8} \\
\infty \\
\infty\end{array}$ & $\frac{n}{\circ}$ & $\begin{array}{l}= \\
\infty \\
\dot{0} \\
i \\
n\end{array}$ & 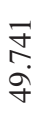 & $\begin{array}{l}\stackrel{\vartheta}{N} \\
\text { in }\end{array}$ & $\stackrel{\circ}{ }$ & $\frac{n}{n}$ & 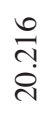 & $\frac{\infty}{\stackrel{\infty}{\stackrel{p}{+}}}$ & $\begin{array}{l}\text { 구 } \\
\infty \\
0 \\
0\end{array}$ & $\begin{array}{l}\stackrel{\circ}{\rightrightarrows} \\
\stackrel{\infty}{+\infty}\end{array}$ \\
\hline $\begin{array}{l}\infty \\
\stackrel{\infty}{0} \\
\text { } \\
\text { గn }\end{array}$ & 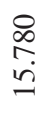 & 0 & 0 & $\begin{array}{l}\hat{a} \\
\hat{0}\end{array}$ & $\begin{array}{l}\tilde{a} \\
\infty \\
0 \\
0 \\
\text { ते }\end{array}$ & $\begin{array}{l}m \\
\infty \\
\stackrel{\sigma}{\sigma} \\
\stackrel{\gamma}{ }\end{array}$ & $\begin{array}{l}\infty \\
\infty \\
\infty \\
\text { ले }\end{array}$ & $\frac{\substack{\infty \\
0 \\
\infty}}{\infty}$ & $\begin{array}{l}n \\
n \\
n\end{array}$ & $\begin{array}{l}\hat{\Xi} \\
\stackrel{\text { I }}{\text { I }}\end{array}$ & $\begin{array}{l}\overline{8} \\
\varnothing \\
\infty\end{array}$ & $\begin{array}{l}\hat{\infty} \\
0 \\
\dot{1} \\
\end{array}$ & $\begin{array}{l}\bar{b} \\
\infty \\
i \\
0\end{array}$ & 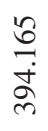 \\
\hline 0 & 0 & 0 & 0 & 0 & $\sigma$. & a. & 0 & $\begin{array}{l}\circ \\
\infty \\
\text { i }\end{array}$ & 0 & 0 & $\frac{n}{n}$ & $\underset{⿱ 亠}{\vec{f}}$ & r. & $\sigma$ \\
\hline 0 & 0 & 0 & 0 & 0 & $\begin{array}{l}n \\
\text { of } \\
\text { nn }\end{array}$ & $\begin{array}{l}\hat{\delta} \\
\hat{n} \\
\dot{y}\end{array}$ & 0 & $\begin{array}{l}0 \\
\text { ஸे } \\
\text { ֻे }\end{array}$ & 0 & 0 & 0 & 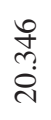 & $\frac{\overparen{I}}{\stackrel{i}{i}}$ & $\frac{\infty}{\stackrel{\infty}{+}}$ \\
\hline 0 & 0 & 0 & 0 & 0 & 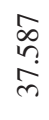 & $\begin{array}{l}\infty \\
\tilde{N} \\
\infty \\
\dot{f}\end{array}$ & 0 & $\underset{⿱}{\mathbb{Z}}$ & 0 & 0 & 0 & 莳 & $\begin{array}{l}\text { N } \\
\infty \\
\infty \\
\infty\end{array}$ & \\
\hline 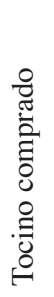 & 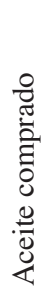 & 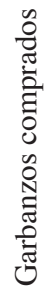 & 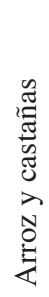 & 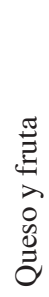 & 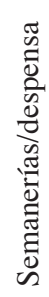 & 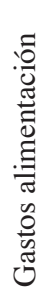 & 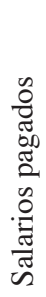 & 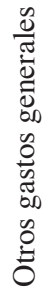 & 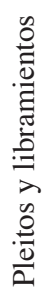 & 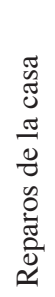 & 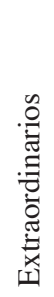 & 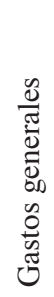 & 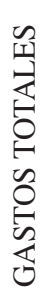 & 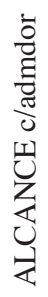 \\
\hline
\end{tabular}

